\title{
Angiotensin II induced the apoptosis of the endothelial progenitor cells by the caspase-3
}

\author{
Yonggang Wu ${ }^{1,2}$, Qiru Wang ${ }^{1}$, Lamei Cheng ${ }^{1}$, Jian Wang ${ }^{1}$, Xuan Sun $^{1}$, Guangxiu Lu ${ }^{1}$ \\ ${ }^{1}$ Institute of Reproductive and Stem Cell Engineering, Central South University, National Center of human Stem Cell Research \\ and Engineering, Changsha, 410078 Hunan, China; ${ }^{2}$ Nuclear medicine Department, Second Xiangya Hospital, Central South \\ University, Changsha 410011, Hunan, China
}

Endothelial regeneration play an important role in restoring vascular tone in respone to vascular injury. And the increased level of angiotensin II (Ang II) is an important risk factor in endothelial injury, which has been considered to play a key role in the initiation and development of atherosclerosis. Endothelial progenitor cells (EPCs) have been shown to enhance the formation of a new endothelim and reendotheliazation. But in previous study, Ang II affects the apoptosis of the EPCs. However, the precise mechanism is still unclear. Therefore, we focused in this study to understand whether Ang II can induce the eNOS (and caspase-3 activity in the EPCs. EPCs were isolated from peripheral blood and characterized. The EPCs were cultured then were treated with Ang II for $48 \mathrm{~h}$. The apoptosis of the endothelial cells was checked by the flow cytometry and, while caspase-3 activity and eNOS was measured simultaneously. After the EPC were treated, the mean of apoptotic rates and caspase-3 enzyme activity and eNOS significantly decreased. Ang II marked inducd a cell cycle at G0/G1 phase and cell apoptosis. and the Angiotension II type1 receptor mRNA is upregulation. therefore Ang II stimulated caspase-3 enzyme leading to apoptosis of the EPCs.

Keywords: EPCs, Ang II, eNOS

Cell Research (2008) 18:s160. doi: 10.1038/cr.2008.250; published online 4 August 2008

Correspondence: Guangxiu Lu

E-mail: lugxdirector@yahoo.com.cn 